Short communication

\title{
Dielectric behavior of porous PMMA: From the micrometer to the nanometer scale
}

\author{
B. Notario ${ }^{\text {a, }}{ }^{*}$, J. Pinto ${ }^{b}$, R. Verdejo ${ }^{c}$, M.A. Rodríguez-Pérez ${ }^{a}$ \\ ${ }^{a}$ Cellular Materials Laboratory (CellMat), Condensed Matter Physics Department, University of Valladolid, Paseo de Belén 7, 47011 Valladolid, Spain \\ ${ }^{\mathrm{b}}$ Nanophysics - Smart Materials Group, Istituto Italiano di Tecnologia (IIT), Via Morego 30, 16163 Genova, Italy \\ ${ }^{\mathrm{c}}$ Institute of Polymer Science and Technology (ICTP-CSIC), Juan de la Cierva 3, 28006 Madrid, Spain
}

\section{A R T I C L E I N F O}

\section{Article history:}

Received 21 December 2015

Received in revised form

31 October 2016

Accepted 14 November 2016

Available online 17 November 2016

\section{Keywords:}

Nanocellular foams

Dielectric properties

Cellular polymers

\begin{abstract}
A B S T R A C T
In recent years, there has been a significant interest of the scientific community on nanocellular polymeric foams as a possible next generation of materials with a low dielectric constant for microelectronics applications In this work, the dielectric behavior of microcellular and nanocellular poly (methyl methacrylate) (PMMA) based foams has been characterized, both as a function of frequency and temperature, in order to analyze the effect of reducing the cell size to the nanoscale on the dielectric properties. Experimental results have shown clear differences in the dielectric behavior of the samples with cell sizes in the nanoscale as well as a sharp reduction of the dielectric constant when the porosity increases.
\end{abstract} () 2016 Elsevier Ltd. All rights reserved.

\section{Introduction}

Nanocellular foams have recently attracted significant attention in the microelectronics industry as a means of producing materials with a low dielectric constant $(k)$ [1-6]. As the devices scale to smaller feature sizes, a new generation of low dielectric constant materials is needed to minimize cross talk and maximize signal propagation speed. The low dielectric constant of polymers and air $(k=1)$ make polymeric foams potential candidates as low $k$ systems [7].

Poly (methyl methacrylate) (PMMA) is a thermoplastic material with a medium to high thermal stability and was one of the first polymers used in microelectronic systems [8]. In fact, this material is frequently used as a dielectric thin film [9-11]. In addition, PMMA has been recently used to produce nanocellular foams with different densities and cell sizes. Due to these reasons PMMA was selected for this study.

In microelectronics, the decreasing dimensions of the devices induces severe size restrictions to the cell size, which should ideally be an order of magnitude smaller than the thickness of the dielectric film [12], i.e. if a polymer foam is used as a dielectric film,

\footnotetext{
* Corresponding author.

E-mail addresses: belen.notario@fmc.uva.es (B. Notario), marrod@fmc.uva.es (M.A. Rodríguez-Pérez).
}

as the thickness of the film could be of a few micrometers the cells should have dimensions in the nanoscale. Therefore, according to the literature, these required cell sizes are merely the result of the dimensions of the devices and it is not expected a significant difference between the well-known dielectric behavior of conventional, or microcellular foams, and that of the recently developed nanocellular foams [13]. However, a preliminary work by the authors has showed the emergence of a Maxwell Wagner Sillars (MWS) phenomenon in PMMA-based nanocellular foams at room temperature [14].

Further research to analyze this unexpected behavior as a function of temperature and frequency could provide important information for the application of nanocellular foams in microelectronic devices [14], where thermal stability is critical and one of the main drawbacks of polymer-based materials [15]. Therefore, the aim of the present study was to investigate in detail the dielectric behavior of both micro and nanocellular foams as a function of frequency and temperature showing the main differences between the behaviors of the two type of materials when cell size is reduced to the nano-scale.

\section{Experimental section}

PMMA was supplied by Arkema Company (France) in the form of pellets. This polymer presents a density $(\rho)$ of $1180 \mathrm{~kg} / \mathrm{m}^{3}$ and a glass transition temperature $\left(T_{g}\right)$ around $112{ }^{\circ} \mathrm{C}$. 
PMMA pellets were injected into pieces of $50 \times 15 \mathrm{~mm}^{2}$ with $3 \mathrm{~mm}$ in thickness (model DSM Xplore), and used later for foaming.

Foaming experiments were performed at room temperature following the solid state foaming process (details can be found in the Supporting Information), modifying the saturation pressure between 10 and $30 \mathrm{MPa}$. The saturation pressures used are included in Table 1.

Dense skin of foamed samples was removed using a polishing machine (model LaboPOl2-LaboForce3, Struers). Then solid and polished samples were machined using a precision cutting machine (Mod. 1000, IsoMet) to be approximately $10 \times 10 \mathrm{~mm}^{2}$ with $1 \mathrm{~mm}$ in thickness. The cellular structure of foams was characterized by scanning electron microscope (SEM, model Quanta 200FEG, FEI).

The dielectric behavior of cellular polymers was evaluated by means of a direct current (DC) electrical resistivity adapter (Keithley 6105, model) and a broadband dielectric spectrometer (ALPHA, Novocontrol Technologies GmbH).

DC resistivity data were determined according to ASTM D257-99 [16]. Solid and foamed samples were measured four times at $+500 \mathrm{~V},-500 \mathrm{~V},+500 \mathrm{~V},-500 \mathrm{~V}$. Time of electrification was $60 \mathrm{~s}$, and the time of discharge before making a measurement with reversed voltage was 4 min. Resistivity $(R)$ was calculated as follows:

$R=\frac{A \cdot V}{t \cdot I}$

where $A$ and $t$ are the area and thickness of the sample respectively, $V$ is the applied voltage, and $I$ is the intensity measured.

In the case of broadband dielectric measurements, foamed and solid samples were held in a dielectric cell between two parallel gold-plated electrodes. The thickness of the samples was taken as the distance between the electrodes and was determined using a micrometer gauge. The complex dielectric permittivity $\left(\varepsilon^{*}=\varepsilon^{\prime}-i \varepsilon^{\prime \prime}\right)$ of the solid and foamed materials was measured over a frequency window of $10^{-2}<\mathrm{F} / \mathrm{Hz}<10^{6}$ ( $\mathrm{F}$ is the frequency of the applied electric field) in the temperature range from -20 to $110{ }^{\circ} \mathrm{C}$. The amplitude of the alternating current (ac) electric signal applied to the samples was $1 \mathrm{~V}$. Furthermore, the real part of the complex dielectric permittivity, $\varepsilon^{\prime}$, (or dielectric constant, $k$ ) was modeled using the series model [17], the parallel model [17], and the Maxwell Garnett model [18].

\section{Results and discussion}

DC resistivity $(R, \Omega / \mathrm{cm})$ at room temperature of PMMA foams with pore sizes between 90 and $3290 \mathrm{~nm}$ (Table 1, representative SEM micrographs of the foams can be found in Fig. S1, see Supporting Information) did not follow a simple trend as a function of to the relative density (Fig. 1, left). The resistivity of the foams increases by two orders of magnitude when the pore size falls from $1460 \mathrm{~nm}$ to $710 \mathrm{~nm}$, despite a simultaneous and slight decrease of the relative density (that should reduce the resistivity).

This behavior was previously observed by J. Pinto and coworkers
[14], who demonstrated the appearance of an electrical conductivity component and an interfacial polarization phenomena (or MWS) in nanocellular PMMA based foams at low frequencies. MWS occurs in heterogeneous materials, such as blends or composites, at the interfaces, leading to a separation of charges (Fig. 1, right). Furthermore, they found an increase in the electrical resistivity of PMMA based foams when cell size shifts from the micro to the nanoscale due to an increase tortuosity of the solid phase (tortuosity of the solid phase is the ratio between the minimum distance of any real path between two areas of the cellular material and the shortest distance between these two areas (Fig. 1, right)).

The appearance of both phenomena can clearly be observed in Fig. 1 left. A clear increase of the resistivity is observed when cell size changes from the micro $(1460 \mathrm{~nm})$ to the nanometer range $(710 \mathrm{~nm})$; this is due to a higher tortuosity of the solid phase in the nanocellular foam (Fig. 1, right). In addition, for materials with cells sizes below $700 \mathrm{~nm}$ a reduction of the resistivity when the cell size is reduced is detected. The reason for this behavior is a conductive mechanism at low frequencies related to the MWS, which is due to the accumulation of charges in opposing cell walls.

As mentioned before, nanocellular polymeric foams are required in the microelectronics due to the reduced thickness of dielectric materials. Therefore, the dielectric behavior of the fabricated PMMA microcellular and nanocellular foams was studied with the aim of understanding if the change in the internal structure can affect the dielectric behavior.

First, the dielectric constant $(k)$ of solid, microcellular and nanocellular PMMA at a frequency of $1 \mathrm{KHz}$ (Table 1 ) was measured. As expected the foams presented reduced $k$ values in comparison with the solid materials. For instance the foam with the smallest pore size $(90 \mathrm{~nm})$ that has a relative density of 0.46 showed an important reduction of $k$ of around 55\% (from 7.73 to 3.56).

With the aim of identifying the possible influence of the pore size in the dielectric constant, the results were compared with the predictions provided by the most common theoretical models used in previous literature. These models consider the system as an isotropic medium and only take into account the fraction of voids (porosity $\left.=\left(1-\rho_{\text {rel }}\right)\right)$ of the foams. It was found that these models fail to predict the experimental results (see Table 1), being not able to simultaneously fit the $k$ values measured for the largest and the smallest pore sizes. This result is probably due to one of the models' hypothesis. In all these models the properties of the constituent phases (both solid and gas phase) do not change with cell size. However previous works [14,19-21], have proved that both the gas within the pores and the polymeric chains in the solid matrix have different properties when the cell size is decreased to the nanometer range. Once these changes are fully understood they should be introduced on more advanced theoretical models able to provide accurate predictions of the dielectric constant for both microcellular and nanocellular foams.

It should be noticed that the values of the dielectric constant of the materials included in this paper are far from the ones currently required by microelectronics industry $(k<2$ [22]). However, the

Table 1

Main characteristics (saturation pressure, cell size and relative density) and, experimental and theoretical values of the dielectric constant ( $k$ ) of the analyzed materials.

\begin{tabular}{|c|c|c|c|c|c|c|}
\hline Saturation Pressure (MPa) & Sample - cell size $(\Phi)(\mathrm{nm})$ & Relative density $\left(\rho_{\text {rel }}\right)$ & $K$ at $1 \mathrm{KHz}$ & $K_{f}$ Series Model [17] & $K_{f}$ Parallel Model [17] & $K_{f}$ Maxwell Garnett Model [18] \\
\hline 0 (Solid) & - (Solid) & 1 & 7.73 & - & - & - \\
\hline 30 & 90 & 0.46 & 3.56 & 1.66 & 4.01 & 3.53 \\
\hline 25 & 200 & 0.49 & 4.25 & 1.74 & 4.28 & 3.73 \\
\hline 20 & 710 & 0.53 & 4.50 & 1.85 & 4.63 & 3.99 \\
\hline 15 & 1460 & 0.56 & 5.07 & 1.95 & 4.89 & 4.19 \\
\hline 10 & 3290 & 0.66 & 5.61 & 2.35 & 5.76 & 4.90 \\
\hline
\end{tabular}



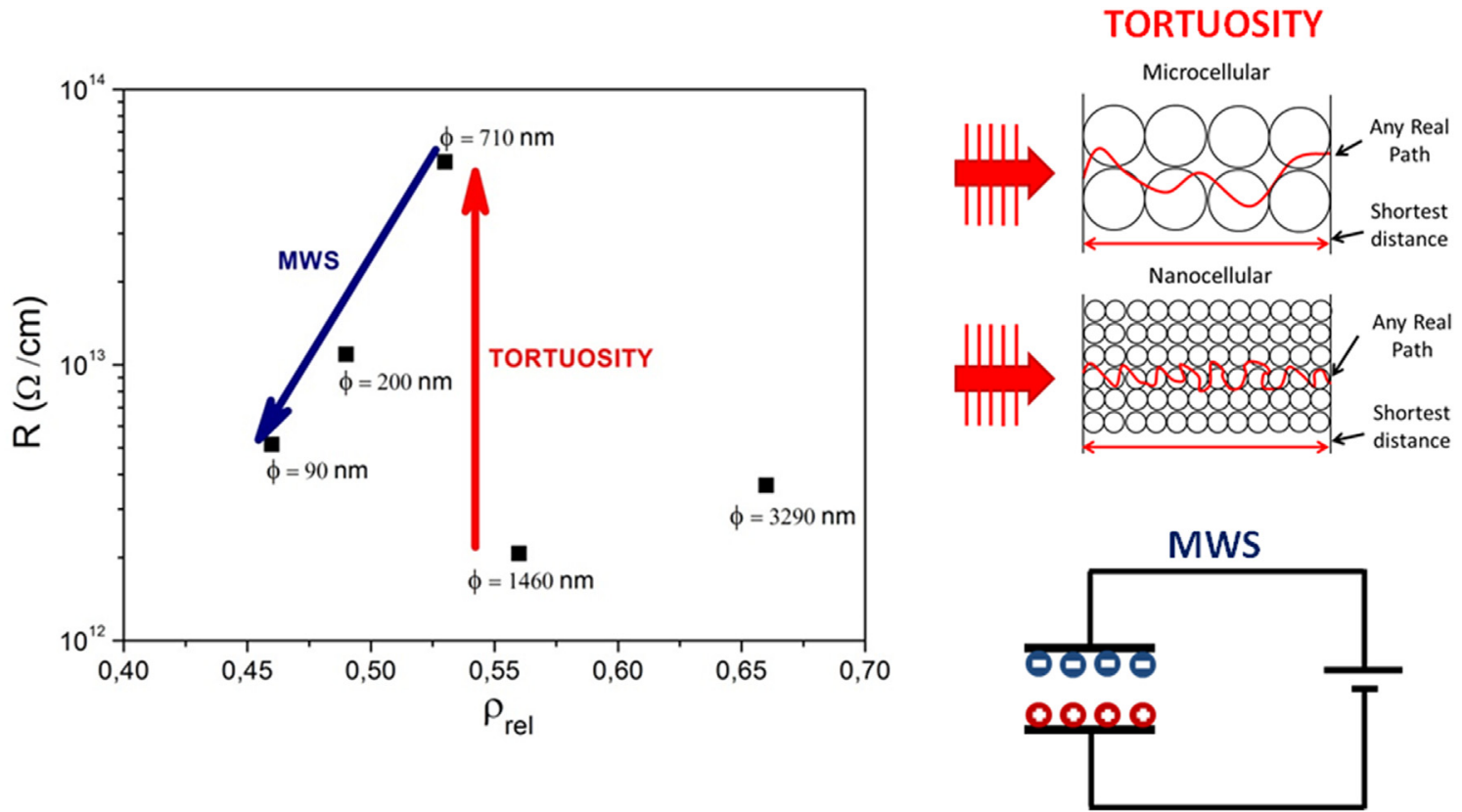

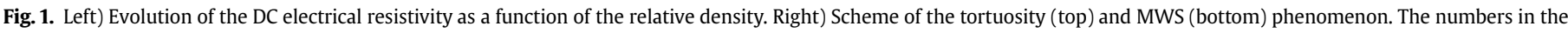
figure of DC resistivity vs relative density correspond to the cell size of each foam.

results presented here show a very promising way of achieving low dielectric constant materials by the production of low density nanocellular foams.

Second,, it was analyzed if the previous evidences of a different behavior of microcellular and nanocellular foams could be confirmed in the frequency domain. With this aim the complex plot of the impedance $(Z)$ or Nyquist plot was obtained for the solid, microcellular and nanocellular PMMA (Fig. 2). Nyquist curves include the entire range of frequencies measured and are used to obtain information about the equivalent electric circuit. In this study a clear change in the samples behaviour is observed. The solid sample presents a dominating capacitor behaviour over the entire frequency range. Nevertheless, since an ideal capacitor appears as a vertical straight line (infinite resistance), the slope that the solid samples presents indicates a slight resistive contribution most likely arising from the capacitor losses present in any dielectric material. This slope then starts to decrease with the presence of the cellular architecture reaching its minimum value in the nanocellular material. Therefore, the nanocellular PMMA develops a

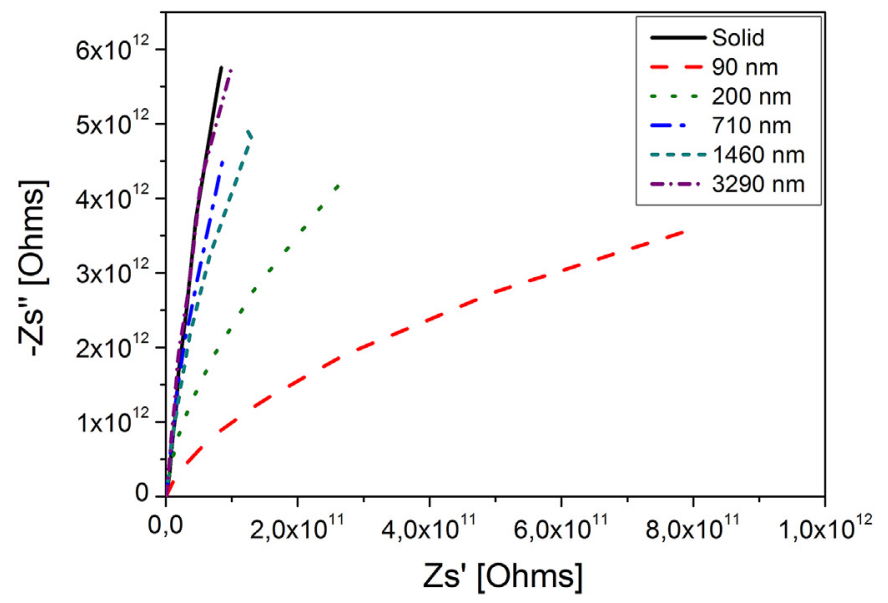

Fig. 2. Nyquist plot for solid, microcellular, and nanocellular PMMA. resistive behaviour and can be modeled as a capacitor in parallel with a resistor. Reduction of the capacitive behaviour found on nanoporous PMMA foams could be related to the progressive immobilization of the polymer chains leading to a progressive decrease of free dipoles able to rotate with the reduction of the pore size.

In short, the previous result confirms that the pore size present a clear influence on the dielectric behavior of PMMA foams. Moreover, dielectric measurements at room temperature confirmed previously published experimental results obtained by measuring the dynamic mechanic behavioral $(\tan \delta$ ) of microcellular and nanocellular PMMA [20]. These results showed that the $\tan \delta$ at low and medium frequencies was increased when the cell size is reduced to the nanometer range (Fig. S2, see Supporting Information).

Finally, the thermal stability of these effects was analysed as a function of the cell size. In order to obtain this information, measurements in the temperature domain were also conducted. In the case of the shape parameters alpha and beta, no differences between microcellular and nanocellular foams were detected as a function of temperature. This behaviour must be further studied due to the previous evidences found in the $\tan \delta$. On the other hand, a decrease of the normalized dielectric strength, $\Delta \varepsilon / \Delta \varepsilon^{\text {solid }}$, that was stable in the temperature range measured (from -20 to $110^{\circ} \mathrm{C}$ ) was observed as a function of cell size (Fig. 3).

This reduction of $\Delta \varepsilon / \Delta \varepsilon^{\text {solid }}$ was previously observed only at room temperature [14] and is was related to a progressive decrease of the number of free dipoles able to rotate, i.e., it is related to the confinement effect of the solid phase in the nanocellular system. Therefore, this results proves that the modifications of the dielectric behavior of PMMA foams induced by the pore size are stable in the temperature domain; a relevant result from an applied point of view.

\section{Conclusions}

The dielectric properties of microcellular and nanocellular PMMA foams have been studied, finding a clear evolution from a 


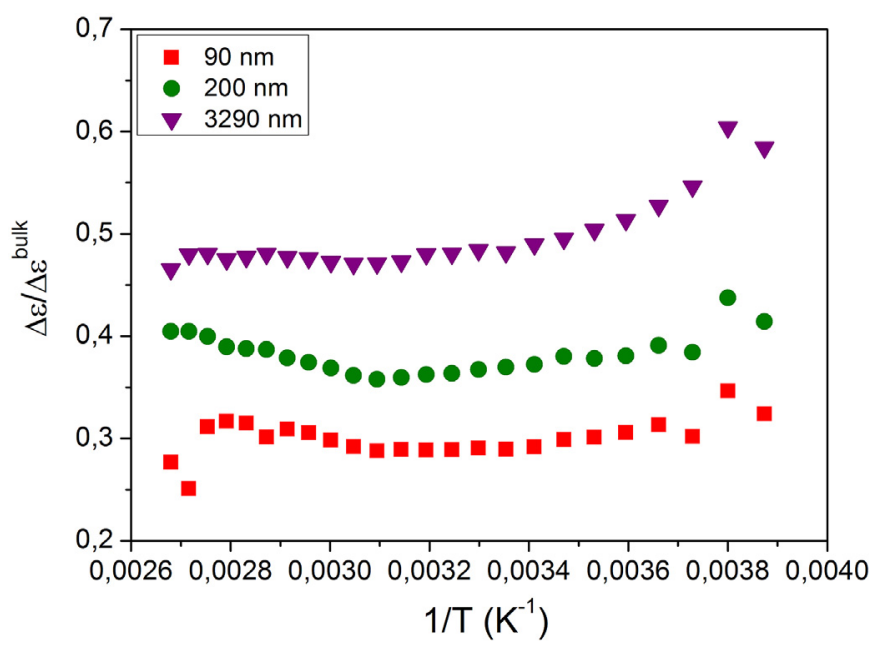

Fig. 3. Evolution of the normalized dielectric strength as a function of the inverse of temperature for materials with different cell sizes.

capacitive behavior to a combination of a resistive and a capacitive response when the cell size is reduced from the microscale to the nanoscale. In particular, it has been found that the dielectric constant of nanocellular PMMA is strongly reduced over that of solid PMMA, showing that the development of nanocellular polymeric materials is a promising way to achieve the next-generation of dielectric materials with a very low thickness (as long as unexpected phenomena like the MWS do not appear at high frequencies). Furthermore, experimental results have confirmed the confinement of the polymer chains along the temperature domain (from -20 to $110^{\circ} \mathrm{C}$ ), showing a great stability up to temperatures close to the glass transition temperature $\left(T_{g}\right)$ of the solid material. Finally, some dynamic mechanical properties previously published of nanocellular materials as well as the dielectric spectrum of PMMA foams have been verified and confirmed [14,20]. Nowadays, the unexpected conductive mechanism found at low frequencies in nanocellular foams is not a problem for the implementation of nanocellular polymeric systems as low dielectric constant materials (since they have to work at high frequencies), but it has to be taken into account to study these systems in more detail and to develop new models to predict its complex behavior.

\section{Acknowledgments}

Financial support from FPI grant BES-2013-062852 (B. Notario) from MINECO and FEDER program (MAT 2012-34901) MINECO, FEDER, UE (MAT2015-69234-R) and the Junta de Castile and Leon (VA035U13) are gratefully acknowledged.

\section{Appendix A. Supplementary data}

Supplementary data related to this article can be found at http:// dx.doi.org/10.1016/j.polymer.2016.11.030.

\section{References}

[1] S. Muruganand, et al., Dielectric and conduction properties of pure polyimide films, Polym. Int. 50 (10) (2001) 1089-1094.

[2] A. Kuntman, H. Kuntman, A study on dielectric properties of a new polyimide film suitable for interlayer dielectric material in microelectronics applications, Microelectron. J. 31 (8) (2000) 629-634.

[3] J.O. Simpson, A.K.S. Clair, Fundamental insight on developing low dielectric constant polyimides, Thin Solid Films 308-309 (1997) 480-485.

[4] Y.H. Zhang, et al., Dielectric properties of polyimide-mica hybrid films, Macromol. Rapid Commun. 26 (18) (2005) 1473-1477.

[5] G.D. Fu, et al., Nanoporous ultra-low dielectric constant fluoropolymer films via selective UV decomposition of poly(pentafluorostyrene)-blockpoly(methyl methacrylate) copolymers prepared using atom transfer radical polymerization, Adv. Funct. Mater. 15 (2) (2005) 315-322.

[6] Y. Xu, et al., Dielectric property and microstructure of a porous polymer material with ultralow dielectric constant, Appl. Phys. Lett. 75 (6) (1999) 853.

[7] R.D. Miller, In search of low-K dielectrics, Science 286 (5439) (1999) 421-423.

[8] X. Peng et al., All-organic thin-film transistors made of alpha-sexithienyl semiconducting and various polymeric insulating layers, Appl. Phys. Lett. 57 (19) (1990) 2013.

[9] J.H. Park, et al., Studies on poly(methyl methacrylate) dielectric layer for field effect transistor: influence of polymer tacticity, Thin Solid Films 515 (7-8) (2007) 4041-4044

[10] K.J. Baeg, et al., Remarkable enhancement of hole transport in top-gated Ntype polymer field-effect transistors by a high-k dielectric for ambipolar electronic circuits, Adv. Mater. 24 (40) (2012) 5433-5439.

[11] A. Berndt, et al., Methacrylate copolymers with liquid crystalline side chains for organic gate dielectric applications, ACS Appl. Mater. Interfaces 7 (23) (2015) 12339-12347.

[12] H.W. Ro, et al., Novel inorganic-organic hybrid block copolymers as pore generators for nanoporous ultralow-dielectric-constant films, Macromolecules 38 (3) (2005) 1031-1034.

[13] B. Notario, J. Pinto, M.A. Rodriguez-Perez, Nanoporous polymeric materials: a new class of materials with enhanced properties, Prog. Mater. Sci. 78-79 (2016) 93-139.

[14] J. Pinto, et al., Molecular Confinement of Solid and Gaseous Phases of Selfstanding Bulk Nanoporous Polymers Inducing Enhanced and Unexpected Physical Properties, submitted for publication, 2016.

[15] D. Shamiryan, et al., Low-k dielectric materials, Mater. Today 7 (1) (2004) 34-39.

[16] ASTM, Standard Test Methods for DC Resistance or Conductance of Insulating Materials, ASTM International, 2005, p. 18.

[17] Y. Wu, et al., Evaluation of mixing rules for dielectric constants of composite dielectrics by MC-FEM calculation on 3D cubic lattice, J. Electroceramics 11 (2003) 227-239.

[18] A. Sihvola, Electromagnetic Mixing Formulas and Applications, The Institution of Electrical Engineers, London, United Kingdom, 1999.

[19] B. Notario, et al., Experimental validation of the Knudsen effect in nanocellular polymeric foams, Polymer 56 (2015) 57-67.

[20] B. Notario, J. Pinto, M.A. Rodriguez-Perez, Towards a new generation of polymeric foams: PMMA nanocellular foams with enhanced physical properties, Polymer 63 (2015) 116-126.

[21] D. Miller, V. Kumar, Microcellular and nanocellular solid-state polyetherimide (PEI) foams using sub-critical carbon dioxide II. Tensile and impact properties, Polymer 52 (13) (2011) 2910-2919.

[22] Association, S.I, National Technology Roadmap for Semiconductors, San Jose, California, 1997. 